Malaria

\section{The genetics}

\section{of resistance}

Lancet 364, 438-447 (2004)

In the borders of Thailand, drug-resistant forms of Plasmodium falciparum - the parasite that causes the most severe form of malaria in humans - are rife. Treatment for malaria in Thailand generally comprises the drugs mefloquine and artesunate, which are reasonably effective when given together. But resistance to this treatment regimen is a problem nevertheless, and resistance to mefloquine alone (the treatment of choice 20 years ago) is very common. The mechanisms of resistance are unclear, however.

Ric N. Price et al. show that the genetics of the parasite plays a part. Over a 12-year period, the researchers studied the responses of 618 malaria patients from Thailand to antimalarial drugs. Patients who were infected with parasites bearing extra copies of a particular gene, called $P$. falciparum multidrug-resistant gene-1 ( $p f m d r 1)$, were most likely to be resistant to mefloquine when it was given alone or in combination with artesunate.

The authors hope that monitoring the number of copies of this gene in the parasites of malaria patients will aid epidemiological surveys of drug resistance in $P$. falciparum. In future, the technique might also be used to predict treatment failure in individual patients. Helen Pilcher

\section{Environmental chemistry}

\section{Blackout bonus}

Geophys. Res. Lett. 31, L13106 (2004)

The power cut of August 2003 may have caused misery for millions of people in the northeastern United States and southeastern Canada, but Lackson T. Marufu and colleagues jumped at the chance it offered to conduct a unique experiment in atmospheric chemistry. More than a hundred power plants were affected by the cuts, and the researchers recognized the unparalleled opportunity to assess the contribution the plants make to air pollution and smog.

The team collected air samples over the blackout area in Pennsylvania, analysing them in real time on board their aeroplane. They compared the results with data taken on the same day just outside the region, and with observations made the previous summer over the same location when meteorological conditions were similar and the power plants were fully operational.

A day into the blackout, ozone had decreased by nearly $50 \%$ and sulphur dioxide was down by $90 \%$. The consequent reduction in sulphate particles, the principal

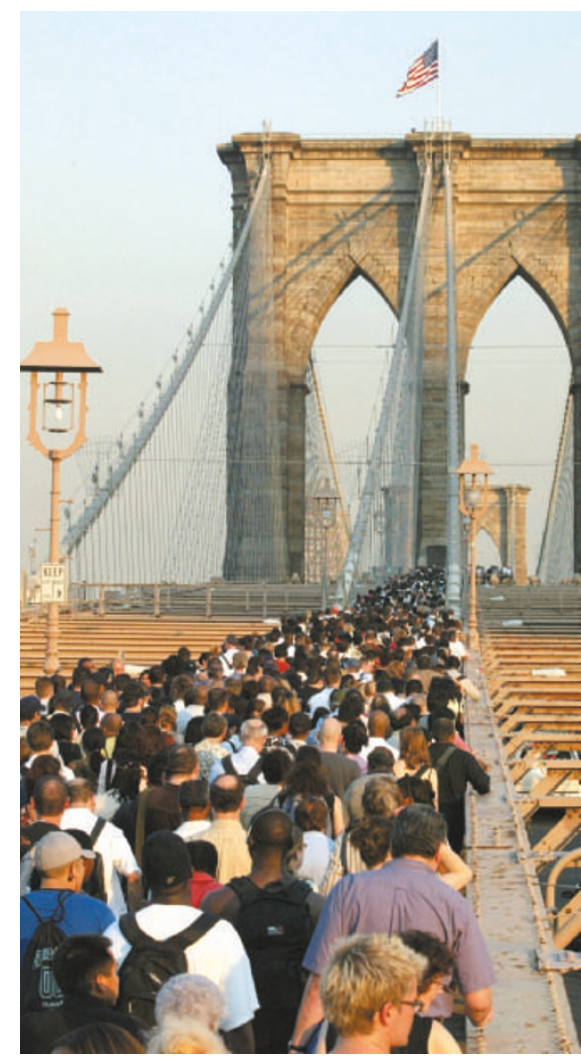

Inconvenient for some: New York in the August 2003 blackout.

cause of haze, meant that visibility improved by more than $40 \mathrm{~km}$. Moreover, much of the eastern United States felt the benefit of the cleaner air, showing that emissions travel hundreds of kilometres from the power stations. The reductions are greater than expected from theoretical estimates, suggesting that models of emissions may have to be rethought.

Helen Dell

\section{Stem cells}

\section{Ready for action}

Cell 118, 149-161 (2004)

Blood cells begin their lives as haematopoietic stem cells (HSCs) inside bone marrow. The bone marrow thus needs a population of these stem cells in a dormant, or quiescent, state, ready to proliferate and differentiate.

Fumio Arai et al. have now unravelled a signalling mechanism that keeps HSCs poised and preserved. Working on bone marrow from adult mice, the authors discovered that HSCs expressing the receptor protein Tie 2 are quiescent, adhere to bone cells and do not die. What's more, the interaction of Tie2 with the angiopoietin-1 protein is sufficient to maintain the long-term blood-repopulating activity of HSCs in vivo.

The authors also point to the recently proposed concept of 'cancer stem cells' — in which cancer cells are replenished by a mechanism rather like the one providing fresh blood cells. They suggest that signalling similar to that seen in HSCs may underpin the ability of leukaemia cells to maintain their populations in the bone marrow, thereby explaining how they can evade chemotherapy. Michael Hopkin

\section{Chemistry \\ Dyeing for cashew nuts}

Ind. Eng. Chem. Res. doi:10.1021/ie030739s (2004)

Fuel oils are routinely dyed to ensure that they are not mixed, diluted or used for purposes other than that allowed under their tax rate. Diesel fuel for agricultural use is often sold at a much lower tax rate, for example, and is dyed to help government officials catch illegal users.

Somsaluay Suwanprasop et al. now report an ingenious new range of fuel dyes made from an agricultural by-product that is ubiquitous in Thailand: the shells of cashew nuts. The shells offer a cheap and plentiful source of cardanol, the starting material for an efficient synthesis of $\mathbf{1 5}$ different dyes. The dyes are normally invisible, but become coloured after a simple extraction procedure. They are also extremely soluble in fuel oils, and can be detected at just five parts per million - a concentration that has no discernible effect on the combustion properties of the fuel, unlike some other marker dyes.

Mark Peplow

\section{Signal transduction}

\section{Flipping the switch}

Mol. Cell 15, 279-286 (2004)

Second messengers, such as cyclic AMP (cAMP) and cyclic GMP (cGMP), are small molecules that help to relay information from the outside to the inside of a cell. They influence a range of processes, including memory, inflammation and the immune response, and are controlled by a family of enzymes called phosphodiesterases. Now Kam Y. J. Zhang et al. have found a molecular switch inside phosphodiesterases that determines which of the second messengers interacts with the enzymes.

The researchers looked at the threedimensional crystal structures of various phosphodiesterases, and found that the orientation of a key glutamine residue determines the enzymes' specificity. In one orientation, this amino acid interacts with cAMP; in another, it interacts with cGMP. In some enzymes, the glutamine residue can flip between orientations, allowing both second messengers to be controlled.

Many medicines, such as Viagra and the anti-inflammatory drug rolipram, target phosphodiesterases. Zhang et al. hope that their discovery will lead to the development of more selective and potent phosphodiesterase inhibitors. Helen Pilcher 